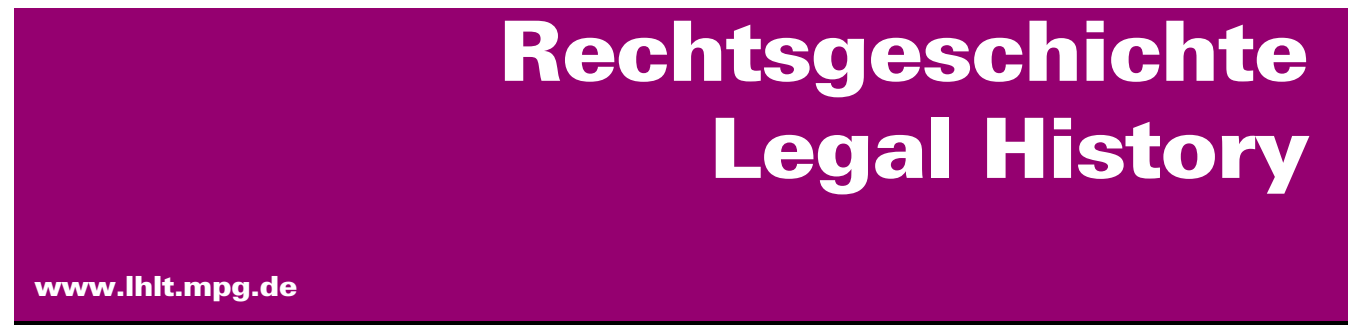

http://www.rg-rechtsgeschichte.de/rg29

$\operatorname{Rg} 292021 \quad 400-405$

Zitiervorschlag: Rechtsgeschichte - Legal History Rg 29 (2021)

http://dx.doi.org/10.12946/rg29/400-405

\title{
Marietta Auer*
}

\section{In weiter Ferne, so nah}

[Faraway, So Close]

* Max-Planck-Institut für Rechtsgeschichte und Rechtstheorie, Frankfurt am Main, auer@lhlt.mpg.de 
Marietta Auer

\section{In weiter Ferne, so nah***}

Harvard - das ist Teil der amerikanischen Popkultur und ein nationaler und globaler Mythos. ${ }^{1}$ An der Geschichte der Harvard University und insbesondere der Harvard Law School, der ältesten kontinuierlich betriebenen Law School der Vereinigten Staaten, lässt sich die politische, juristische, intellektuelle und soziale Geschichte Amerikas der vergangenen zwei Jahrhunderte wie durch ein Brennglas studieren. Seit ihrer Gründung im Jahr 1817 hat sie zwei amerikanische Präsidenten, siebzehn Richter des Supreme Courts, zahlreiche Träger weiterer höchster Staatsämter sowie einen erheblichen Teil der Professorenschaft an amerikanischen Law Schools hervorgebracht. Die Autoren, ausgewiesene Spezialisten der Universitätsgeschichte sowie der amerikanischen Geschichte, begründen ihr monumentales Unterfangen, die Geschichte der Harvard Law School in zwei jeweils gut 600 bzw. 800 Seiten starken Bänden darzustellen, daher zu Recht mit dem »Isomorphismus« der amerikanischen Rechtsausbildungskultur, der immer wieder nach Harvard zurückverweist (4ff.). Dieser Begriff beschreibt die eindrucksvolle Leistung der Harvard Law School, in der Juristenausbildung über zwei Jahrhunderte hindurch maßstabsetzend gewirkt zu haben, und zwar in nahezu allen Bereichen vom Fächerkanon über die Lehrmethode bis hin zum Wirtschaftsmodell der Juristenfakultät. Der hier rezensierte zweite Band des Gesamtwerks umspannt die acht Jahrzehnte vom Dekanat Ezra R. Thayers (19101915) bis zu den politischen Fakultätskämpfen der 1980er Jahre. Glänzend geschrieben und mit reichem Bild- und Zahlenmaterial ausgestattet, erweckt er das Harvard des 20. Jahrhunderts so anschaulich zum Leben, dass man die weißen Kirchturmspitzen, roten Backsteingebäude und goldenen Herbstfarben Neuenglands selbst dann buchstäblich vor Augen sieht, wenn man sie nicht wie die Autorin dieser Zeilen tatsächlich kennenlernen durfte.

Je länger man sich mit dem rezensierten Band befasst, desto klarer tritt dabei zutage, dass die Juristenausbildung dies- und jenseits des Atlantiks viel mehr unvermutete Gemeinsamkeiten als Unterschiede aufweist. Das gilt bereits für das erwähnte Wirtschaftsmodell, und zwar erstaunlich unabhängig davon, ob man eine privat finanzierte amerikanische Spitzenuniversität wie Harvard oder die chronisch unterfinanzierte Juristenfakultät einer deutschen Massenuniversität betrachtet. Es gilt weiter für die Lehrmethode, den Fächerkanon, die Wissenschaftlichkeit oder Praxisrelevanz der Ausbildung sowie für die während des gesamten 20. Jahrhunderts nicht abreißende Debatte über juristische Ausbildungsreformen. Doch die Liste ist noch länger: Sie umfasst die Bedeutung von Benotungssystemen und meritokratischen Selektionsmechanismen für die Verteilung juristischer Karriere- und Lebenschancen. Sie umfasst weiter eine Studienkultur, in der Angst und Leistungsdruck eine signifikant größere Rolle spielen als in den Ausbildungskulturen anderer Fächer. Und sie umfasst schließlich den Habitus des durch rigorose akademische Auslese geformten, zu Führungsaufgaben aller Art befähigten modernen Berufsjuristen, dessen Prägung mit der Selbstselektion eines bestimmten Studierendentypus beginnt und sich durch normalisierende Karrierepfade bis hin zur hochselektiven Elite der auf Lebenszeit berufenen Universitätsprofessoren laufend selbst reproduziert. Wiederum ragt dabei das strukturkonservative Beharrungsvermögen der juristischen Fakultäten auf beiden Seiten des Atlantiks gleichermaßen aus dem akademischen Fächerdurchschnitt heraus.

\footnotetext{
* Bruce A. Kimball, Daniel R. Coquillette, The Intellectual Sword. Harvard Law School, The Second Century, Cambridge (MA)/London: The Belknap Press of Harvard University Press 2020, 880 S. ISBN 978-0-674-73732-7
}

** Ich widme diesen Beitrag meinem Lehrer Duncan Kennedy zum 80. Geburtstag im Jahr 2022 in Dankbarkeit für alles, was ich von ihm an der Harvard Law School auch und gerade über Juristenausbildung lernen durfte.
1 Einen kleinen Überblick über die seit dem 19. Jahrhundert erschienenen Harvardiana bietet der erste Band des hier rezensierten Werks: Bruce A. Kimball, Daniel R. Coquillette, On the Battlefield of Merit. Harvard Law School, The First Century, Cambridge (MA) / London 2015, 7-12. 
Ein Beispiel: Zu den eindrucksvollsten Erträgen des rezensierten Bandes zählt die Mikrogeschichte der jahrzehntelang verschleppten Öffnung der Harvard Law School für Frauen und Minderheiten. Diese Verschleppung betraf alle Ebenen der Institution von den Studierenden über die Professoren bis zum Verwaltungspersonal und konnte sich nicht trotz, sondern gerade wegen des ausgeprägt meritokratischen Selbstverständnisses der Law School, in deren männlich-weiß dominiertem Milieu Juristenausbildung ausdrücklich auch als Charakterbildung nach einem Ideal spartanischer Härte und Männlichkeit verstanden wurde, besonders lange und hartnäckig halten. Für alles, was dem dazugehörigen WASP-Korpsgeist nicht entsprach, war an der Harvard Law School bis weit in die zweite Hälfte des 20. Jahrhunderts hinein kein Platz. Den Autoren gelingt es meisterlich, diese langlebige Dynamik in historischen Querschnitten unter Rückbezug auf den persönlichen Stil und biographischen Hintergrund der jeweils amtierenden Dekane und Universitätspräsidenten sowie eines großen Kreises von weiteren Akteuren und Betroffenen $\mathrm{zu}$ veranschaulichen. So bedeutete Minderheitendiskriminierung während des langen Dekanats von Roscoe Pound (1916-1936) unter dem Präsidium des Bostoner Bilderbuch-Brahmanen Abbott Lawrence Lowell (1909-1933) etwas anderes als in den 1950er und 1960er Jahren unter Dekan Erwin Griswold (1946-1967) sowie den Präsidenten James Conant (1933-1953) und Nathan March Pusey (1953-1971); und abermals ganz anders stellte sich die Situation während der 1970er und 1980er Jahre unter der Ägide von Derek C. Bok (Dekan 1968-1971, Präsident 1971-1991) sowie den Dekanen Albert M. Sacks (1971-1981) und James Vorenberg (1981-1989) dar.

Unter Pound und Lowell blieb es unhinterfragter Konsens, Frauen die Zulassung zum Studium an der Harvard Law School zu verwehren, obwohl sich die Fakultät bereits zu Beginn des 20. Jahrhunderts mehrfach für ihre Zulassung ausgesprochen hatte und es in den folgenden Jahrzehnten immer wieder Anläufe qualifizierter Frauen gab, diese Hürde zu überwinden ( 29 ff. u. ö.). Die Stärke des Werks liegt darin, die selbstreproduzierende akademische Kultur Harvards, die solche Aufbrüche trotz des guten Willens vieler Protagonisten über Jahrzehnte hinweg blockierte, plastisch herauszuarbeiten. Paradigmatisch kommt diese institutionelle Kultur etwa in einer von den Autoren zitierten Stellungnahme Lowells aus dem Jahr 1915 zum Ausdruck, als dieser auf die Frage eines Pressevertreters nach den Gründen für die Ablehnung einer Petition von fünfzehn Frauen, zu gleichen Bedingungen wie Männer zum Jurastudium zugelassen zu werden, sinngemäß antwortete, Harvard könne keine Frauen zulassen, weil die Zulassung Männern vorbehalten sei (31). Nach dem Zweiten Weltkrieg war diese Argumentationslinie allerdings nicht mehr durchzuhalten. $\mathrm{Zu}$ diesem Zeitpunkt war Harvard als eine von nur noch fünf Law Schools in den gesamten Vereinigten Staaten, die Frauen den Zugang verweigerten, längst in eine Außenseiterposition geraten. Also hieß die Law School im Herbst 1950 sang- und klanglos den ersten Studienjahrgang von vierzehn Frauen auf dem Campus willkommen, nachdem sich Fakultät und Universität unter dem Technokraten Conant 1949 endlich auf deren Zulassung geeinigt hatten und die Fakultät das Zulassungsverfahren anschließend wegen "mangelnder Erfahrung « mit Frauencolleges monatelang verschleppt hatte (457 ff.). Wieder liefert der Band auch diesseits des Atlantiks reichhaltiges Anschauungsmaterial dafür, was Diskriminierung von Frauen und Minderheiten in der Rechtswissenschaft jenseits des Etiketts dieses schnell ausgesprochenen oder hingeschriebenen Begriffs eigentlich bedeutet: Unter minutiöser Aufbereitung des verfügbaren Zahlenmaterials schildern die Autoren, wie die Zahl der Studentinnen an der Law School bis in die 1960er Jahre hinein stets nur einen Bruchteil der zugelassenen Männer pro Jahrgang ausmachte, weil so das ständig wiederholte Argument - vermieden werden müsse, dass Frauen qualifizierte Männer von Studienplätzen verdrängen, zumal nicht zu erwarten sei, dass sie in den erlernten juristischen Berufen lange tätig sein würden. Einmal zugelassen, hatten die wenigen Jurastudentinnen im Harvard der 1950er und 1960er Jahre Ordalien zu bestehen wie Einladungen zum Dinner bei den Griswolds, wo jede einzelne von ihnen peinlich befragt wurde, was sie bewogen habe, an die Law School zu kommen und einem Mann den Studienplatz wegzunehmen (469 ff.), oder »Ladies Days« in den Vorlesungen einiger notorischer Professoren, bei denen die Frauen, die sonst übergangen wurden, vor den vollbesetzten Hörsaal zitiert und zum Gaudium der männlichen Zuhörerschaft über »Frauenthemen« wie Ehescheidung oder häusliche Gewalt examiniert wurden (467 f.). Erinnert sich jemand an die hierzulande geführte 
Debatte über stereotype Geschlechterrollen in juristischen Ausbildungsmaterialien ${ }^{2}$

Doch die Frauen reüssierten, und einige $\mathrm{Na}$ men überragender Alumnae wie Ruth Bader Ginsburg tauchten, nicht viel anders als die der besten männlichen Alumni, zeitversetzt ab den 1970er Jahren wieder an der Harvard Law School auf, diesmal als Kandidatinnen für die Besetzung von Professuren (477 ff., 695 ff.). Doch mit jeder bezwungenen Karrierestufe wiederholte sich das Elend sexualisierter sozialer Exklusion: Hatten es die Frauen erst in die elitäre "Harvard Law Review« geschafft, erwies sich der weitere Weg zur Professur als versperrt, der über die prestigeträchtigen wissenschaftlichen Mitarbeiterstellen am Supreme Court führte (515 ff., 524). Hatte sich dieser Weg einmal geöffnet, sahen sich die in Harvard angekommenen Professorinnen mit ungreifbaren sozialen Hürden konfrontiert, die vom »lonely lunch syndrome« $(701,703)$ über vorgebliche Probleme mit der Lehre bis hin zu endlos verschleppten Tenure-Entscheidungen reichten (692 ff.). Die präzise aufbereitete Zeittafel der in den 1970er und 1980er Jahren begutachteten und abgelehnten oder besser von der »spartanischen Kultur« vertriebenen Kandidatinnen für TenurePositionen an der Harvard Law School liest sich wie ein Who's Who bedeutender amerikanischer Juristinnen des 20. Jahrhunderts und stellt der Institution ein Armutszeugnis ersten Ranges aus (811 f.). Auch hier gelingt es den Autoren, die am Werk befindlichen sozialen Praktiken an konkreten Einzelschicksalen plastisch hervortreten zu lassen, ohne historische Komplexitäten durch nachgereichte Bewertungen zu reduzieren. So gehen etwa bis heute die Einschätzungen der für den Band eigens befragten Zeitzeuginnen darüber auseinander, ob Dekan Griswold den studierenden Frauen jenseits der abweisenden institutionellen Kultur persönlich wohlgesonnen war oder nicht (469 ff.). Viele weitere eindrucksvolle Geschichten dieser Art könnten hier noch erwähnt werden:
Die der offen praktizierten Zurücksetzung jüdischer Studierender und Rechtswissenschaftler in den 1920er Jahren, bei der der spätere NS-Sympathisant Pound und der WASP-Gentleman-Jurist Lowell am selben Strang zogen (173 ff., $178 \mathrm{ff}$., 253 ff., 797 f.). Die des Kampfes afroamerikanischer Studierender und Rechtswissenschaftler um Gleichberechtigung und berufliche Anerkennung, der erst nach der Bürgerrechtsbewegung der 1960er Jahre Früchte trug (182 f., 480 ff., 643 ff., 690 ff., 706 ff.). Schließlich die der Kommunistenjagd unter Studierenden und Professoren, die in den 1920er Jahren in der aufgeheizten Atmosphäre des Sacco-Vanzetti-Prozesses erstmals ihr hässliches Gesicht zeigte und im McCarthyismus der 1950er Jahre in jakobinischen Terror ausartete, der Karrieren und Leben zerstörte und gegen den selbst die juristische Spitzenkultur der Harvard Law School kaum etwas ausrichten konnte (134 ff., 406 ff.).

Die von den Autoren erzählte Geschichte der Harvard Law School ist jedoch mehr als ein großer Bilderbogen der amerikanischen Gesellschaftsgeschichte und Juristenbiographik. Sie ist auch Rechtswissenschaftsgeschichte, Institutionengeschichte, Fakultätsgeschichte und vor allem nach Amtsperioden übersichtlich geordnete Dekanatsgeschichte, die den Leser immer wieder mit den Fragen konfrontiert: Wie steuert man eine Institution wie die Harvard Law School durch ein Jahrhundert voller politischer Umbrüche? Welche Reformen waren nötig und wurden realisiert, welche Entwicklungen hingegen verschleppt oder verschlafen? Welche finanziellen und personellen Risiken darf und muss man eingehen, um die eigene Spitzenposition zu halten, und wie prekär darf es finanziell höchstens werden? Die überraschende Antwort der Autoren lautet, dass das Wirtschaftsmodell der Law School deutlich prekärer war, als es ihr nach außen zur Schau gestellter Reichtum, ihre zahlreichen exzellenten Studenten und ihre durch das ganze Jahrhundert hindurch
2 Die hier wie dort nur einen kleinen Ausschnitt des Geschlechtseffekts in der Juristenausbildung abbildet; vgl. Daniela Schweigler, Das Frauenbild in der bayerischen Justizausbildung, in: DRiZ 2014, 52-55 sowie weitergehend EMANUEL Towfigh, Christian Traxler, ANDREAS GLÖCKNER, Zur Benotung in der Examensvorbereitung und im ersten Examen, in: ZDRW 1 (2014)

8-27; Phillip Hellwege, Julia

DORFSCHMIDT, KaTHaRINA SCHAR-

Rer, Martina Benecke, Frauen in

den Rechtswissenschaften, in: RW 6

(2015), 301-353; Thomas Hinz,

Hans Christian RöHL, Geschlech-

terunterschiede in der Ersten juristischen Prüfung, in: JZ 71 (2016), 874-880. 
vorangetriebene üppige Bau- und Expansionspolitik vermuten ließen. Die Autoren konstatieren ein "tuition dependence syndrome", hinter dem ein über Jahrzehnte hinweg selbstverstärkender Rückkopplungskreislauf zwischen struktureller Unterfinanzierung, großen Jahrgangsstärken und dadurch erzwungener weiterer Expansion der Law School steckt: Aus struktureller Unterfinanzierung folgte die Notwendigkeit, möglichst viele Studierende zuzulassen, um den Betrieb der Law School durch die laufenden Einnahmen aus Studiengebühren zu decken. Für die vielen Studierenden waren Campus und Fakultät aber chronisch unterdimensioniert, so dass immer weiter expandiert werden musste, was wieder neues Geld kostete, das mangels Kapitalreserve nur durch noch mehr Studierende erwirtschaftet werden konnte - und so weiter (105 ff., 216 ff., 569 ff. u. ö.). Fühlt sich jemand an das Finanzierungsmodell der hiesigen Juristenfakultäten erinnert, die ihre typische Drittmittelschwäche im Gesamtplan der Hochschulfinanzierung etwa im Vergleich mit den medizinischen Fakultäten ebenfalls nur durch überproportionale Kapazitätsauslastung wettmachen können, was dazu führt, dass Hörsäle überbelegt, Betreuungsverhältnisse schlecht und Abbrecherquoten hoch sind? So etwas wie Drittmittelschwäche gab es in der ersten Jahrhunderthälfte auch in Harvard, wo Dekane wiederholt an der Aufgabe scheiterten, Alumni und reiche Geldgeber zu Spenden an die Law School und nicht etwa an die im naturwissenschaftlichen 20. Jahrhundert viel einleuchtender nützliche Medical School zu bewegen ( $347 \mathrm{ff}$.). Und noch in einer anderen Hinsicht zeigen die Autoren anschaulich, dass Juristenausbildung ein Wirtschaftsunternehmen ist und die Basis hier wie dort den Überbau bestimmt: Die für den Band titelgebende Kultur des »intellektuellen Schwerts«, die bis in die zweite Jahrhunderthälfte hinein in übertrieben feinen Notendifferenzierungen (34 ff., 529 ff., 664 ff. u. ö.) und Durchfallquoten von bis zu einem Drittel im ersten Studienjahr zum Ausdruck kam, war nicht zuletzt direkte Folge der Abhängigkeit von Studiengebühren und einer institutionellen Politik, die jahrzehntelang lieber hohe Einschreibezahlen und hohe Durchfallquoten akzeptierte als auf die Studiengebühren der später aussortierten Studenten zu verzichten $(9,788$ u. ö.). Sogar zu dem hierzulande nach wie vor geläufigen Angstmacherspruch aus Erstsemesterveranstaltungen, wonach von den beiden Sitznachbarn bei einem Blick nach links und rechts am Ende des Jahres einer nicht mehr da sein werde, gab es in Harvard einst ein exaktes Pendant (160).

Was hätte man an der Harvard Law School, an der diese Zeiten heute längst vorbei sind, schon früher anders und besser machen können? Die Law School hätte, wie die Autoren für jede Zeitschicht und jedes Dekanat einzeln darlegen, aus der Abhängigkeit von Studiengebühren ausbrechen können, wenn sie rigoros auf das namentlich von der Yale Law School seit den 1920er Jahren erfolgreich als Gegenentwurf zu Harvard praktizierte Boutique-Modell hochselektiver Zulassung, geringer Jahrgangsstärken, kleiner Klassen, wissenschaftlicher Spezialisierung und daraus folgender konstant kleiner, aber exzellent besetzter Fakultät sowie geringer Bautätigkeit umgeschwenkt wäre (586 ff.). Wäre das, wie die Autoren nahelegen, auch ein für Harvard gangbarer Weg gewesen? Die meisten Dekane der Law School waren anderer Ansicht, so namentlich Griswold, der diese Überzeugung 1950 in die schlichten Worte kleidete, seine Vorgänger hätten entschieden, dass Harvard eine große Law School sein solle (515, 553). Nun: Wer es zwei Jahrhunderte lang schafft, die maßgebliche Isohypse für den von den Autoren konstatierten Isomorphismus der amerikanischen Juristenausbildung zu setzen, kann so viel nicht falsch gemacht haben. Und so verfolgt man gespannt, wie die einzelnen Dekane im Spagat zwischen Law School, Universität und Zeitgeschichte der Aufgabe gerecht wurden, die weitreichenden und wagemutigen Investitionsentscheidungen $\mathrm{zu}$ treffen, die die Spitzenstellung der Harvard Law School bis heute prägen. Nicht alle waren dieser Verantwortung gewachsen; manche zerbrachen an ihr. Thayer (1910-1915) beging Selbstmord, Pound (1916-1936) mutierte zum Faschisten, und James M. Landis (1937-1946) zog eine Skandalspur ehebrecherischer Verhältnisse und Steuerdelikte nach sich, ehe er am Alkohol zugrunde ging (21 ff., 253 ff., 291 ff.). Indessen hatte die zukunftsweisende Expansion bereits unter James Barr Ames (1895-1910) mit dem Bau der Langdell Hall begonnen, die als Sitz der weltgrößten rechtswissenschaftlichen Bibliothek bis heute das Gesicht der Law School prägt ( 73 ff., 213 ff.). Wäre es eine Alternative gewesen, dieses Gebäude nicht zu errichten und stattdessen im Baubestand des 19. Jahrhunderts mit einer auf diese Maße eingefrorenen Fakultät und Studentenschaft zu verharren? Wohl kaum. Noch deutlicher wird die insti- 
tutionelle Fruchtbarkeit des zukunftsgerichteten Wachstums am Dekanat Griswolds (1946-1967), durch dessen Initiative nicht nur weitere große Bauprojekte realisiert wurden, sondern der auch darüber hinaus für viele zukunftsweisende Reformen von der deutlichen Vergrößerung und Modernisierung der Fakultät über die Einführung eines professionellen Alumni-Managements bis hin zur Abkehr von der Politik überzähliger Zulassungen und hoher Durchfallquoten verantwortlich zeichnete ( 378 ff.). Seitdem betreibt Harvard bekanntermaßen eine ebenso selektive Zulassungspolitik wie Yale, nur auf höherem Zahlenniveau. Insgesamt zeigt das Dekanat Griswolds, was gutes Hochschulmanagement in der Juristenausbildung bewirken kann - wieder eine unbedingt lesenswerte Lektion auch und gerade diesseits des Atlantiks.

Den Nachfolgern Bok (1968-1971), Sacks (1971-1981) und Vorenberg (1981-1989) fiel die Aufgabe zu, die Law School durch die von politischen Grabenkämpfen geprägten Jahrzehnte von der Bürgerrechtsbewegung bis zur Reagan-Ära zu steuern. Seit den 1960er Jahren hatte sich das gesellschaftliche Klima in den Vereinigten Staaten grundlegend gewandelt. Dabei spielten sich einige der signifikantesten Veränderungen wiederum auf der Mikroebene des sozialen Systems der Law School ab. Ein Beispiel bildet der Niedergang der bis in die 1960er Jahre wichtigsten Frauenorganisation auf dem Campus, der "Harvard Law Wives" (462 ff.). Diese bot den Ehefrauen studierender Männer seit den 1930er Jahren ein Forum zum sozialen Austausch, wobei die Gattin des Dekans als Schirmherrin fungierte und die selbstlose Unterstützung der Karriere der studierenden Männer im Vordergrund stand. Doch mit diesem Idyll dienender Weiblichkeit war es vorbei, als der spätere Dekan Bok auf einem Treffen der Vereinigung im Jahr 1960 dafür warb, dass Frauen sowohl im eigenen als auch im Interesse ihrer Männer ihre eigene Karriere verfolgen sollten. Die empörten Reaktionen zeigten nur, dass das Frauenbild auch auf dem Campus der Harvard Law School längst in unaufhaltsamem Wandel begriffen war. Die dort seit zehn Jahren studierenden Frauen hatten kein Interesse an den Treffen der Harvard Law Wives, weil sie sich in deren Rollenverständnis nicht wiedererkannten. In den 1970er Jahren löste sich die Vereinigung schließlich auf. Auch diese kleine Erzählung erweist sich als exemplarische Mikrogeschichte, die den breiten Verfall hergebrachter sozialer Rollen und Autoritäten seit den 1960er Jahren sogar im konservativen Milieu der Law School illustriert. Die symbolische Macht der alten Ordnung wirkte nicht mehr: Erratisches Verhalten von Professoren, zu Griswolds Zeiten noch an der Tagesordnung (382 ff.), war nunmehr verpönt; aus Klagen über harte Studienbedingungen wurden politisch ausgetragene "grade wars" (664 ff., 734 ff.); Frauen und Minderheiten forderten lautstark Gleichbehandlung; die elitäre WASP-Clubkultur starb aus (741); wo früher durchregiert wurde, musste jetzt langwierig diskutiert werden. Unterdessen explodierten die Studiengebühren mitten in die Rezession der 1970er Jahre hinein (683 ff.).

Am Ende des Bandes steht die durch fakultätspolitische Auseinandersetzungen rund um das "Critical Legal Studies Movement« zerrüttete Fakultät der 1970er und 1980er Jahre (763 ff.). Aus heutiger Sicht wirkt dessen linksradikale Politik gesellschaftlichen Wandels durch Enthierarchisierung der Law School indessen geradezu erfrischend. Dabei ging es noch um ein echtes politisches Ziel, nämlich um die Partizipation bislang ausgeschlossener Gruppen. Die Rollen von Freund und Feind waren klar verteilt, als Duncan Kennedy 1980 sein »Utopian Proposal on Law School as a Counterhegemonic Enclave« formulierte und dabei nicht weniger als eine grundlegende Reform des Zulassungsverfahrens, des Benotungssystems, der Auswahl der Mitglieder der Law Review, der Professorenberufung und Professorenbesoldung forderte, um »the School's role in promoting illegitimate hierarchies « zu reduzieren oder umzukehren (730, 770 ff.). ${ }^{3}$

Man spürt allerdings von ferne schon den Frosthauch politischer Überempfindlichkeit, der seit-
3 Grundlegend und bis heute lesenswert Duncan Kennedy, Legal Education and the Reproduction of Hierarchy: A Polemic Against the System, Cambridge (MA) 1983, neuveröffentlicht: New York 2004. Vgl. bereits Ders., How the Law
School Fails: A Polemic, in: Yale Review of Law \& Social Action 1 (1970) 71-90; DERs., First Year Law Teaching as Political Action, in: Law \& Social Problems 1 (1980) 47-58. 
dem in den amerikanischen Hochschulen Einzug gehalten hat und mit der üblichen Zeitverzögerung inzwischen unübersehbar auch in Europa angekommen ist. Am Ende des Bandes wird deutlich, wie sehr in der gegenwärtigen akademischen Cancel Culture die Saat aufgegangen ist, die durch die gesellschaftliche Polarisierung der Vereinigten Staaten seit der Reagan-Ära gesät worden ist. Natürlich darf dabei auch die Geschichte des Wappens der Law School nicht fehlen, das 2016 unter internationaler Medienaufmerksamkeit abgeschafft wurde, nachdem die Autoren im ersten Band ihrer hier vorgelegten Geschichte den Zusammenhang zwischen dessen Gestaltung in

\section{Amber Rose Maggio Heimatlos*}

Mira L. Siegelbergs Monographie Statelessness: A Modern History ist eine umfassende Begriffsgeschichte der Staatenlosigkeit als Konzept und als rechtliches Phänomen. Wie die Autorin im einleitenden Kapital schreibt, bezieht sich das Konzept der Staatenlosigkeit auf eine breite Palette von Fragen rund um die politische Organisation der Menschheit, umfasst gleichzeitig aber einige der destabilisierendsten Entwicklungen der modernen Politik. Siegelberg untersucht diese Themen und Entwicklungen anhand einer Vielzahl von Quellen und legt so eine Ideengeschichte der Staatenlosigkeit vor, die zum Kern der Frage vordringt, wie die politische Organisation um Souveränität dem Menschenrechtsschutz für Staatenlose widerspricht.

Wie man es von einer Geschichte eines Rechtsbegriffs erwarten würde, sind viele der in dem Band herangezogenen Quellen juristischer Natur. Sie reichen von nationalen und internationalen Gerichtsentscheidungen, den Minderheitenverträgen nach der Pariser Friedenskonferenz, den Berichten und Dokumenten des Völkerbundes und
Anlehnung an das Familienwappen Isaac Royalls, der den ersten Lehrstuhl an der Law School gestiftet hatte, und der Herkunft des Stiftungsvermögens aus dessen von Sklaven betriebener Zuckerplantage in Antigua hergestellt hatten (2 ff., 394 ff.). Wieder handelt es sich um ein signifikantes Stück Mikrogeschichte, in der sich die große Zeitgeschichte spiegelt, und man möchte der Harvard Law School wünschen, dass sie noch viele Jahrzehnte und Jahrhunderte in ständiger Transformation auf ihrer einzigartigen Höhenlinie ausharrt, wo der Wind des Wandels rauer weht als anderswo. den Akten der Haager Kodifizierungskonferenz 1930 bis hin zur nationalen Gesetzgebung und natürlich den völkerrechtlichen Verträgen. Zugleich zeichnet dieses geistesgeschichtliche Werk das politische Denken in der turbulenten Zwischenkriegszeit über den Zweiten Weltkrieg bis in die Nachkriegszeit nach, unter besonderer Berücksichtigung von Rechtstheoretikern und Emigranten. Interessanterweise greift die Autorin auch auf fiktionale Werke zurück, die versuchen, die Erfahrung derjenigen einzufangen, die in dem Zustand leben, keinen Staat zu haben: heimatlos zu sein. Die reiche Vielfalt an Quellen und die Auseinandersetzung mit dem Gedanken der Staatenlosigkeit auf unterschiedlichen Ebenen liefern eine originelle und aufschlussreiche Darstellung, die über die bisherige Forschung zu diesem Thema hinausgeht.

Staatenlosigkeit als Kategorie definiert sich durch ihre fehlende Verbindung zum Staat. Siegelbergs Darstellung der Entwicklung dieses Konzepts zeigt, wie wichtig die Vorstellung von staatlicher Souveränität für die Positionierung von

\footnotetext{
Mira L. Siegelberg, Statelessness:

A Modern History, Cambridge (MA) /

London: Harvard University Press

2020, 318 p., ISBN 978-0-674-97631-3
} 